Please do not remove this page

RMIT

UNIVERSITY

\title{
Adaptive region growing impulse noise estimator for color images
}

Phu, Meing; Tischer, Peter; Wu, Henry

https://researchrepository.rmit.edu.au/esploro/outputs/9921860286701341/filesAndLinks?institution=61RMIT_INST\&index=null

Phu, M., Tischer, P., \& Wu, H. (2006). Adaptive region growing impulse noise estimator for color images. Proceedings of The 18th International Conference on Pattern Recognition Volume 3, 786-789.

https://doi.org/10.1109/ICPR.2006.223

Published Version: https://doi.org/10.1109/ICPR.2006.223

Repository homepage: https://researchrepository.rmit.edu.au

(C) 2006 IEEE

Downloaded On 2023/04/26 22:40:45 +1000

Please do not remove this page 


\section{Adaptive Region Growing Impulse Noise Estimator for Color Images}

\author{
Mieng Quoc Phu, Peter Eric Tischer \\ Clayton School of Information Technology, \\ Monash University, Vic, Australia. \\ \{mieng.quoc.phu,peter.tischer\}@.csse.monash.edu.au
}

\author{
Hon Ren $\mathrm{Wu}$ \\ Software and Network Engineering, \\ RMIT University, Vic, Australia. \\ henry.wu@rmit.edu.au
}

\begin{abstract}
In this paper, a novel region growing impulse noise estimator for color images is proposed. The aim of this estimator is to distinguish noisy pixels from uncorrupted pixels and subsequently measure the noise proportion efficiently. We use a region growing technique to segment the images into clusters of pixels and propose an adaptive decision scheme to measure the noise proportion. Performance analyses show the proposed scheme outperforms some of the state-of-theart techniques.
\end{abstract}

\section{Introduction}

In the area of image and video processing, impulse noise corrupts images either by hardware or natural phenomenon such as thunder and lightning. The corrupted images not only look unpleasant but also make it difficulty for any preprocessing task in the area of image processing, such as image compression, detection process and data analysis.

Over the last decade, many filters have been proposed for natural color image restoration [1-6]. Their aims are to reconstruct an image resembling the original and minimizing the mean square error. State of the art filters such as adaptive vector median filter (AVMF) [1], selection center-weighted vector directional filter (SCWVDF) [2] and self adaptive algorithm (SAA) [3] are some of the most efficient switch based filters. They rely on the impulse detectors to classify clean pixels from corrupted pixels. These impulse detectors can be used to estimate the amount of noise in the image.

Recently, as in the SAA filter, noise filters require an estimate of the proportion of noise-affected pixels in the image. Noise filters need to determine the amount of noise in the image before any detection and reconstruction process. This will reduce the inevitable misclassification of pixels.
In this paper, we proposed a fast impulse noise estimator for random impulsive noise. The proposed ARGIE (adaptive region growing impulse estimator) is based on the region growing approach to segment the entire image and then an ADS (Adaptive Decision Scheme) is used to determine the noise proportion.

This paper is organized as follows. Section 2 describes the structure of the proposed ARGIE. Section 3 shows the effects of the threshold parameter. In Section 4 simulations and discussions are presented and finally in section 5 the conclusions.

\section{The proposed ARGIE}

Figure 1 shows the proposed structure of the proposed ARGIE. It includes the region growing detection scheme (RGDS) and the ADS.

Let us define $I, S$ and $B$ as the input, cluster and binary image, respectively. This scheme creates a binary image $B$, showing whether a pixel is corrupted or not. For each pixel, the entry in the $\mathrm{S}$ shows to which cluster the pixels belongs and there are $\mathrm{K}$ clusters in $I$. We further define $s_{k}$ be the number of pixels in clusters $C_{k}$ in image $S$, where $\mathrm{k}$ is the identification number of each cluster in $S$. For example given an image $I$, we have

$$
\bigcup_{i=1}^{K} C_{i}=I, \quad C_{i} \bigcap_{i \neq j} C_{j}=0
$$

\subsection{The RGDS}

In this approach, the input image is segmented into clusters of similar pixel intensity. The method used is known as region growing. In region growing, only one pixel is added to a 'spatially coherent' cluster at a time. Spatially coherent means that only adjacent pixels are allowed to form clusters. For example, if we start with 
a cluster of one pixel, then the neighbouring pixels are examined in turn. If the neighbouring pixel is sufficiently closed, i.e. the equation (2) is satisfied for two adjacent pixels where $\delta$ a threshold and $\mathrm{N}$ is the number of neighbouring pixels, and it has not already been added to any cluster, it is added to the cluster and we then consider neighbours of that pixel. If we have run out of neighbours for the pixel which was most recently added, we return to the pixel added before that and continue to examine its neighbours. The process continues until all the pixels in a given image have been examined. Notice, the cluster of size $s_{k}$ has a range of $\left\{1 \leq s_{k} \leq H \times W\right\}$, where $H$ is height and $W$ is the width of the image. If every pixel in the image has at least one neighbour whose value is sufficient close, then every pixel will be in the one cluster $\left(s_{1}=W \times H\right)$. However, the existence of impulse noise may mean that there are pixels in clusters with only a small number of elements. In general, uncorrupted pixels will be in clusters with large numbers of elements. These two remarks are the essence of our proposed technique. The algorithm of the RGDS can be implemented recursively.

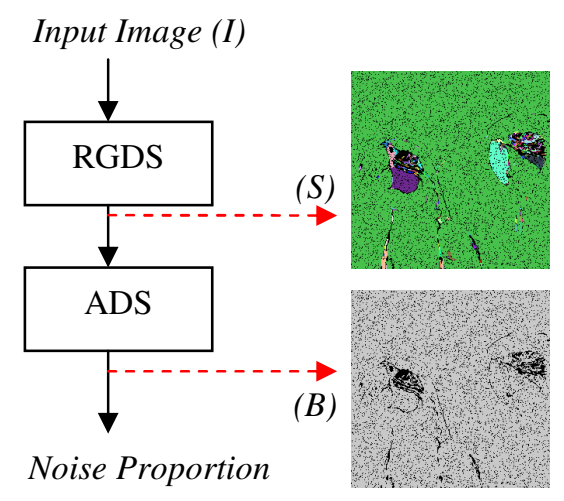

Figure 1: The proposed ARCHIE structure. Random colors are assigned to $S$ image to differentiate clusters.

\subsection{The ADS}

Once the RGDS process is finished, the ADS process determines which clusters are corrupted. Because impulse noise is randomly distributed over the whole image, if the proportion of corrupted pixels $(\phi)$ is less than $50 \%$, it is rare that a cluster containing only corrupted pixels has more than 6 members. Our process assumes the pixels in clusters which have 7 members are not corrupted. In addition, if a cluster contains only one member, we assume this pixel must be corrupt.
Thus, if we assume the pixels of the binary image, $B$, take either the value 1 to represent 'noisy pixel' or the value 0 to represent a 'clean pixel', then, the rules (3) and (4) always apply when estimating impulse noise for $\phi \leq 50 \%$ corruption..

For clusters with 2, 3, 4, 5 and 6 members we can classify them as being corrupt or clean depending on the proportion of pixels believed to be corrupted. Thus, we must adaptively adjust the condition dependent on the estimated noise proportion $\phi_{e}$. From the data we collected on the randomness of impulse noise, we have formulated an adaptive approach to determine whether a cluster consists purely of noisy pixels. This switch based approach is shown in (5) and (6), where $s_{\max }$ is the maximum cluster size to be classified as 'noisy'. Using the decision rules in (5) and (6), all pixels can be classified as being either corrupted or clean and this can be stored in a binary image. To compute $\phi_{e}$ we used the equation (7). We assume that all pixels in a cluster with only 1 or 2 members are corrupted and we use the total number of pixels in these clusters to determine an estimated proportion. $\phi_{e}$ is a good initial estimator but as noise increases this estimate worsens because the number of noise clusters which have more than 2 members increases proportionally to noise.

$$
\begin{gathered}
\left\|x_{i}-x_{j}\right\|_{2}<\delta \quad j \in\{1, \ldots, N\} \\
\text { If } s_{k} \geq 7, \quad \forall x \in C_{k}, \quad x \text { is '}^{\prime} \text { clean' } \\
\text { If } s_{k}=1, \quad \forall x \in C_{k}, \quad x \text { is 'noise' } \\
s_{\max }= \begin{cases}1 & \phi_{e}<0.9 \% \\
2 & 1 \leq \phi_{e}<9.6 \% \\
3 & 9.6 \leq \phi_{e}<23 \% \\
4 & 23 \leq \phi_{e}<31 \% \\
5 & 31 \leq \phi_{e}<39 \% \\
6 & \phi_{e} \geq 39 \%\end{cases} \\
\text { If } s_{k} \leq s_{\max }, \quad \forall x \in C_{k}, \quad x \text { is 'noise' } \\
\phi_{e}=\frac{\varphi}{W \times H} \times 100, \quad \varphi=\sum_{s_{k} \leq 2} s_{k}
\end{gathered}
$$




\section{The effects of the threshold $\delta$}

From section 2.1 the threshold $\delta$ from equation (2) is very important. The effects of $\delta$ on the cluster image $S$ and on $B$ are shown in Figure 2. Random colors are assigned to $\mathrm{S}$ image to differentiate clusters for display purpose. For small values of $\delta$, more clusters are formed and the number of small clusters increases significantly. This occurs mainly at the edges due to many variations of pixel intensity. Thus, the RDGS often misclassifies clean pixels as noisy pixels. In contrast, for large values of $\delta$, fewer clusters with more pixels are formed. This in effect will misclassify noisy pixels as being clean pixels. From our study, we found that the compromise value of $\delta$ should be in the range of 35 to 60 for natural color images. In this paper, we used 40 for the proposed ARGIE in both the Lena and Parrots images.

\section{Simulation and discussion}

The impulse noise corruption for color images is modeled as by [1-3]. Denote $Q_{i}$ and $x_{i}$ as the corrupted pixel and original pixels, respectively. Let $p$ be the noise proportion, and then the impulse noise corruption given in (8).

$$
x_{i}=\left\{\begin{array}{l}
Q_{i} \quad \text { with probability } p \\
x_{i} \quad \text { with probability } 1-p
\end{array}\right.
$$

Because of channel correlation in color images, a two-step impulse corruption is employed. First, the given image is corrupted in each channel independently with a random value of range $[0,255]$. Then a factor $\rho=0.5$ is used to introduce more noise into the other color channels for each corrupted pixel. In other words, there is a $50 \%$ chance of further corruption if one channel has already been corrupted. In practical terms, the actual corruption in a given image is always less than the user input noise proportion due to channel correlation corruption overlapping and the redundancy of randomness value. The degree of redundancy depends on the input noise percentages.

\subsection{Noise proportion assessments}

In this section the color images of Lena and Parrot of size 256x256 are corrupted with random impulse noise. The noise proportion is ranging from 0 to $50 \%$. The 'actual noise' is the proportion of pixels values that actually changed. Table 1 and 2 show the performance of the proposed estimator ARGIE compared with those used by the state-of-the-art filters AVMF [1], SCWVDF [2] and SAA [3]. All parameters setting are implemented as recommended by the referenced authors.

The aim of this experiment is to see which estimator can efficiently estimate the noise proportion in a given image. The estimator that produces results which are closest to the 'actual noise' proportion is the most efficient. From Table 1, for the Lena image, the proposed ARGIE outperforms SAA for all noise percentages and AVMF for all noise percentages except for the Parrot image at 5\% from Table 2. Compared to the SCWVDF, ARGIE did well for most percentages. In addition, as the noise proportion increases ARGIE outperform all estimators significantly, up to $4 \%$ improvements. These remarks also reflected in the Parrots image in Table 2. Moreover, from the results, it can be seen that all estimators include the proposed ARGIE tend to underestimate the noise proportion. This is because of the redundant errors, results from random impulse noise sometimes blend into the image structure making them very difficulty to detect.

\section{Conclusion}

A novel and simple random impulse noise estimator is proposed in this paper. The scheme used global region growing technique and a newly proposed adaptive decision scheme to detect impulse noise and subsequently used to estimate the noise proportion. The results showed that it is robust and very efficient for various noise percentages.

\section{References}

[1] R. Lukac, Adaptive vector median filtering, Patt. Recogn. Lett. 24 (2003) 1889-1899.

[2] R. Lukac, Adaptive color image filtering based on centerweighted vector directional filters, Multichannel Sys. Signal Process. 15(2) (2004) 169-196.

[3] B. Smolka, et al., Self-adaptive algorithm for impulsive noise reduction in color images, Patt. Recogn. 35(8) (2002) 1771-1784.

[4] J. Astola, P. Haavisto, Y. Neuov, Vector median filter, Proc. IEEE 78(4) (1990) 678-689.

[5] E. S. Hore, B. Qiu, H. R. Wu, Prediction based image restoration using a multiple window configuration, Optical Engineering, Vol. 41, (Aug. 2002) 1-11.

[6] C. Kenney, et al., Peer group image enhancement, IEEE Trans. Image Processing, 6(7), (Feb 2001) 326-334. 

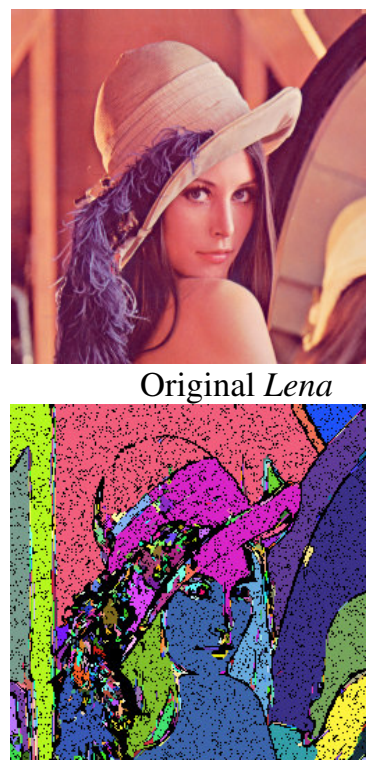

S image $(\delta=10)$

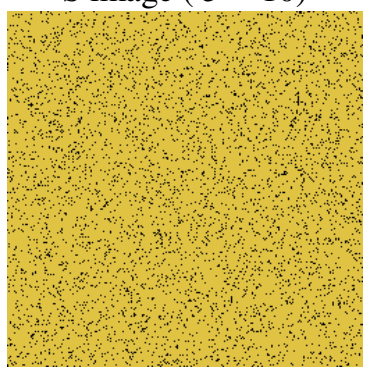

S image $(\delta=60)$

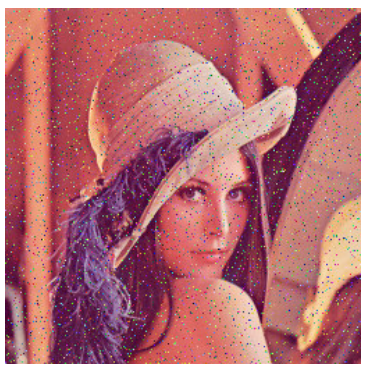

$10 \%$ Noise

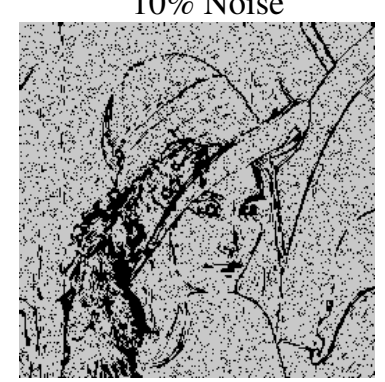

B image $(\delta=10)$

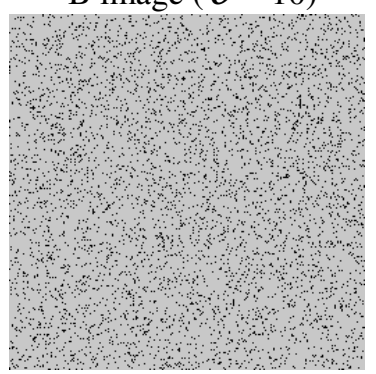

B image $(\delta=60)$

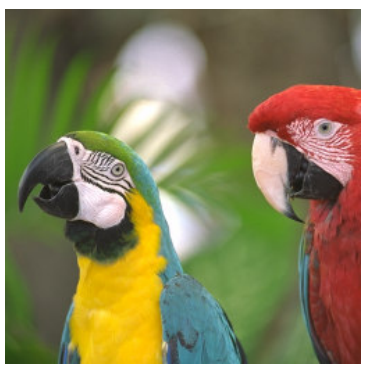

Original Parrots

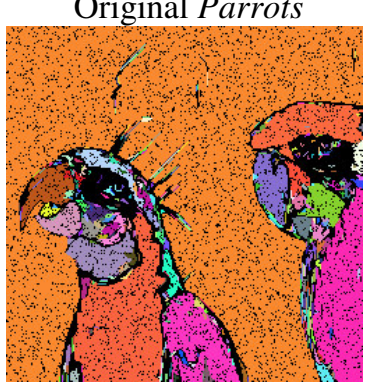

S image ( $\delta=10$ )

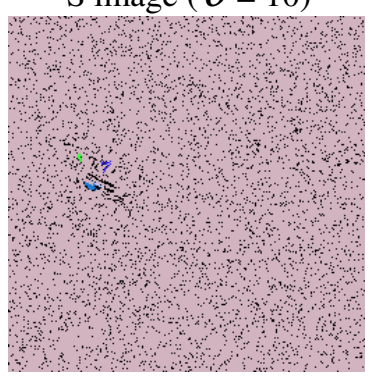

S image ( $\delta=60$ )

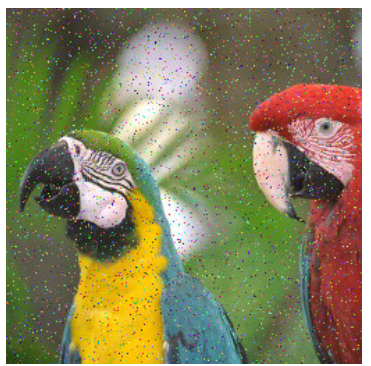

$10 \%$ Noise

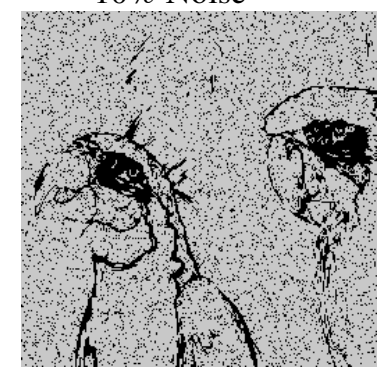

B image ( $\delta=10)$

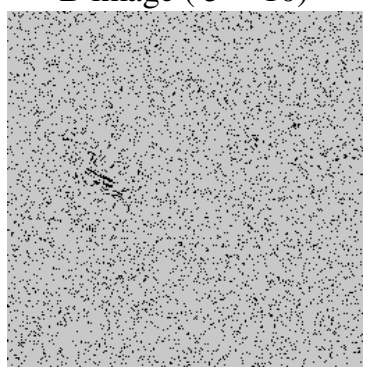

B image $(\delta=60)$

Figure 2: The effects of varying $\delta$ on the $10 \%$ corrupted image of Lena and Parrots.

Table 1: The performance on Lena of different estimators compared with ARGIE for various Random noise proportions.

\begin{tabular}{|c|c|c|c|c|c|c|c|c|c|c|c|}
\hline \multirow{2}{*}{$\begin{array}{l}\text { Estimators/ } \\
\text { Detectors }\end{array}$} & \multicolumn{11}{|c|}{ USER INPUT NOISE PROPORTION (\%) } \\
\hline & $\mathbf{0}$ & $\mathbf{5}$ & 10 & 15 & 20 & 25 & 30 & 35 & 40 & 45 & $\mathbf{5 0}$ \\
\hline Actual Noise & 0.000 & 4.898 & 9.781 & 14.545 & 18.860 & 23.038 & 27.010 & 31.302 & 35.030 & 38.553 & 42.293 \\
\hline AVMF [1] & 0.453 & 4.332 & 8.252 & 12.061 & 15.610 & 18.810 & 22.011 & 25.404 & 28.467 & 31.136 & 34.187 \\
\hline SCWVDF [2] & 0.311 & 4.739 & 9.084 & 13.199 & 16.850 & 20.198 & 23.376 & 26.540 & 29.161 & 31.404 & 33.777 \\
\hline SAA [3] & 0.137 & 3.935 & 7.726 & 11.278 & 14.369 & 17.052 & 19.641 & 23.738 & 26.335 & 27.972 & 30.281 \\
\hline ARGIE & 0.262 & 4.771 & 9.007 & 13.074 & 16.907 & 20.477 & 24.234 & 28.035 & 31.825 & 34.926 & 38.495 \\
\hline
\end{tabular}

Table 2: The performance on Parrot of different estimators compared with ARGIE for various Random noise proportions.

\begin{tabular}{|c|c|c|c|c|c|c|c|c|c|c|c|}
\hline \multirow{2}{*}{$\begin{array}{c}\text { Estimators/ } \\
\text { Detectors }\end{array}$} & \multicolumn{11}{|c|}{ USER INPUT NOISE PROPORTION (\%) } \\
\hline & $\mathbf{0}$ & 5 & 10 & 15 & 20 & 25 & 30 & 35 & 40 & 45 & $\mathbf{5 0}$ \\
\hline Actual Noise & 0.000 & 4.866 & 9.480 & 14.256 & 18.787 & 22.766 & 26.935 & 31.013 & 35.251 & 38.522 & 42.435 \\
\hline AVMF [1] & 0.977 & 4.826 & 8.553 & 12.303 & 15.771 & 19.174 & 22.408 & 25.589 & 29.031 & 31.528 & 34.749 \\
\hline SCWVDF [2] & 0.098 & 4.663 & 8.897 & 13.255 & 17.067 & 20.525 & 23.914 & 27.080 & 30.101 & 32.524 & 35.141 \\
\hline SAA [3] & 0.159 & 3.891 & 7.762 & 11.438 & 14.586 & 17.456 & 20.178 & 22.559 & 26.913 & 28.882 & 30.939 \\
\hline ARGIE & 0.354 & 5.234 & 9.351 & 13.260 & 17.142 & 20.880 & 24.680 & 28.192 & 32.356 & 35.295 & 39.043 \\
\hline
\end{tabular}

